\title{
Electron Capture in Collisions of Slow Highly Charged Ions with an Atom and a Molecule: Processes and Fragmentation Dynamics
}

François Frémont, Guillaume Laurent, Jimmy Rangama, Przemyslaw Sobocinski, Medhi Tarisien, Lamri Adoui, Amine Cassimi, Jean-Yves Chesnel, Xavier Fléchard, Dominique Hennecart and Xavier Husson

Centre Interdisciplinaire de Recherche Ions Lasers, Unité Mixte CEA-CNRS-ISMRA-Université de Caen Basse Normandie, 6 Bd du Maréchal Juin, F-14050 Caen Cedex, France

Tel.: +33 (0) 2314525 67, Fax: +33 (0) 2314525 57, E-mail: francois.fremont@ismra.fr

Received: 9 October 2001/Accepted: 22 November 2001/ Published: 28 March 2002

\begin{abstract}
Processes involved in slow collisions between highly charged ions (HCI) and neutral targets are presented. First, the mechanisms responsible for double electron capture are discussed. We show that, while the electron-nucleus interaction is expected to be dominant at projectile velocities of about 0.5 a.u., the electron-electron interaction plays a decisive role during the collision and gains importance when the projectile velocity decreases. This interaction has also to be invoked in the capture of core electrons by HCI. Finally, the molecular fragmentation of $\mathrm{H}_{2}$ following the impact of $\mathrm{HCI}$ is studied.

Keywords: Electron capture, highly charged ions, neutral target, electron-electron interaction, molecular fragmentation.
\end{abstract}

\section{Introduction}

One of the most important processes that occur in collisions between highly charged ions (HCI) and atoms or molecules at low velocity is the electron capture or charge transfer. Beyond the fundamental aspects, the study of electron capture is of great interest in various research areas such as astrophysics, controlled thermonuclear fusion or ion accelerator technology [1]. For example, it has been known for decades that charge exchange occurs between solar wind ions and neutral gas in the interstellar medium, but it is only very recently that the resulting X-ray emission from HCI in the wind has been 
considered [2,3]. These X-ray observations are fundamental since they are connected to the knowledge of stellar wind parameters, such as mass loss, wind velocities and ion composition, which are needed to constrain models of stellar evolution [3].

From a general point of view, slow collisions involving HCI are characterized by at least two parameters, i.e. the impact velocity $v_{\text {proj }}$ and the number of target electrons. The electron capture process is dominant if $v_{\text {proj }}$ is smaller than the classical velocity of the target electrons. The impact velocity, which determines the duration of the collision and thus, the adiabaticity of the process, has a strong influence on the collision dynamics. Consequently, the strength of the different dynamic interactions are modified when varying $v_{\text {proj. }}$. Hence, the final states of the projectile and the associated cross sections are also modified. Similarly, the number of target electrons as well as their binding energies influence the collision dynamics by increasing the number of reaction pathways and the variety of inelastic processes.

For single electron capture (SEC), energy gain spectroscopy [4], photon spectroscopy [5] and recoil ion momentum spectroscopy (RIMS) [6,7] have been used extensively to measure with good accuracy total, partial and differential cross sections. In parallel, theoretical methods, such as the classical overbarrier (COB) model [8], the Landau-Zener model [9], the classical trajectory Monte Carlo (CTMC) model [10], and molecular expansion close-coupling methods [11] have been developed. From such experimental and theoretical works, the main features have been explained successfully over a wide range of impact velocities [1,12].

The situation is different for multiple electron capture. This is due to the fact that the number of active electrons involved is larger, leading to a more complex, many-body problem. A further difficulty lies in the high number of transient molecular states necessary to describe the collision. In order to reduce these difficulties, experiments [13-15] and calculations [11,16,17] were devoted to double electron capture (DEC) from a helium target (two active electrons). The helium atom is an interesting target because it is easily prepared in collision experiments and its electronic structure is the simplest one for a theoretical treatment of double capture.

However, even in the case of collisions involving only two active electrons, the processes responsible for DEC are still under debate [12,18-21]. While DEC can be rather well described using independent electron models, it has been shown that dynamic correlation effects due to the electronelectron interaction which is not incorporated in these models may play a decisive role during the collision [22,23]. The importance of these processes depends strongly onto the collision systems involved [24], and the projectile velocity [15,18].

Over the last few years, much work has been devoted to the study of slow collisions between HCI and molecules [25-31]. The study of ion-molecule collisions is fundamental since it gives information not only on the electronic processes involved during the collision, but also on the dynamics of the molecular fragmentation that occurs after the collision. While a complete treatment of such collisions appears impossible, due to the high number of collision partners involved, it has been shown that the collision can be divided into two independent steps, since the collision time (typically $10^{-16} \mathrm{~s}$ ) is much shorter than the typical vibrational time in the molecule $\left(\sim 10^{-12} \mathrm{~s}\right)$. The first step is the collision itself, where the capture processes occur. The second step is the fragmentation of the residual ionized target. Consequently, the many-body problem to solve can be simplified. However, the description of the whole collision requires the knowledge of the capture processes, which is the main goal of ion-atom collision studies. 
After the collision, the projectile may decay via photon emission or Auger electron emission. Since the doubly excited states involved during the collision decay mainly via Auger electron emission, the experiments were performed using the method of Auger electron spectroscopy [13,18,22-24]. Recently, more detailed information was obtained using imaging techniques and Recoil Ion Momentum Spectroscopy (RIMS) [6,32]. These techniques were shown to be powerful methods to investigate ion-atom and ion-molecule collisions [6,32,33].

In this paper, the mechanisms responsible for electron capture are discussed. First, collision systems involving fully stripped ions and a He target are investigated for collision velocities from 0.6 a.u. down to 0.01 a.u., using the methods of Auger electron spectroscopy and RIMS. The present measurements extend our previous experimental studies performed for $\mathrm{C}^{6+}$ and $\mathrm{Ne}^{10+}+\mathrm{He}$ collisions. The relative importance of the capture mechanisms is analyzed, as a function of the projectile charge and velocity. Then, the fragmentation of $\mathrm{H}_{2}$ molecules following the impact of $\mathrm{O}^{5+}$ projectiles is analyzed. In particular, it is shown that the two-step picture invoked above is no longer valid at the very low impact velocities involved in our experiments.

\section{Ion-atom collisions - Double electron capture mechanisms}

\section{Electron capture mechanisms}

The mechanisms responsible for DEC are illustrated in Figure 1 which shows approximate orbital energies for the system $(\mathrm{C}-\mathrm{He})^{6+}$. In the incident channel, two electrons occupy the He $1 s$ orbital that crosses the $2 \ell$ and $3 \ell$ orbitals of $C$ at internuclear distances of $\sim 2$ and 6 a.u., respectively. Hence, the DEC process may occur by two independent one-electron transitions (denoted monoelectronic in the following), due to the nucleus electron interaction. Then, configurations of quasi equivalent electrons $2 \ell 3 \ell^{\prime}$ and $3 \ell 3 \ell^{\prime}$ are produced $[22,23]$.

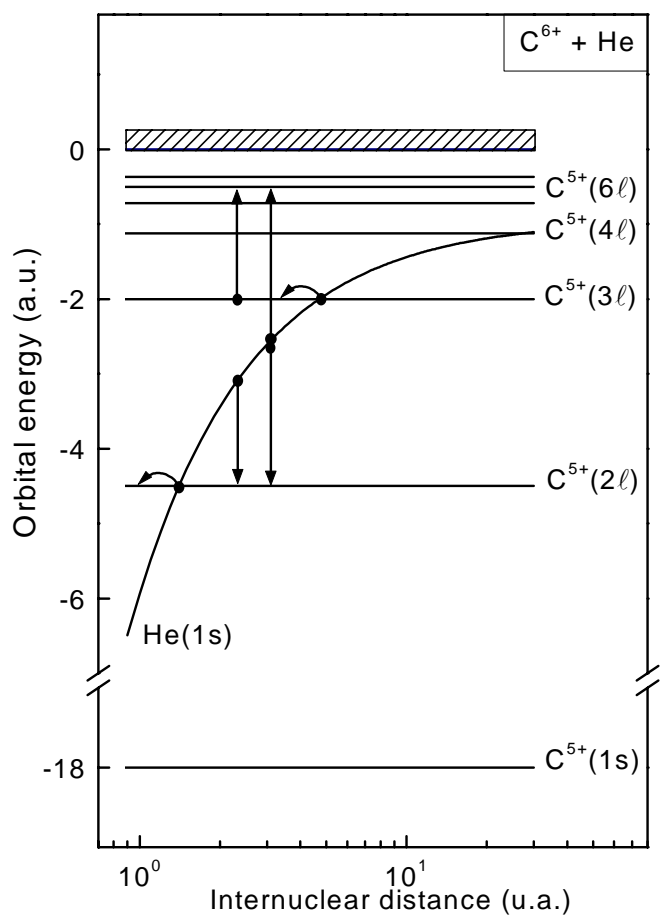

Figure 1. Diagram of orbital energies for the system $(\mathrm{C}-\mathrm{He})^{6+}$. 
Furthermore, as shown in Figure 1, resonance conditions are created at $\sim 3$ a.u. for a dielectronic process referred to as correlated double capture (CDC) process, due to the electron-electron interaction. The CDC process produces configurations of non equivalent electron $n \ell n$ ' $\ell^{\prime}(n$ ' > $n$ ), such as $2 \ell 6 \ell^{\prime}$ in Figure 1. It is pointed out that two independent one-electron transitions are unlikely to produce such configurations, since the probability to populate the $6 \ell$ orbital by a monoelectronic transition is negligible.

A correlated two-step mechanism may also lead to the production of the configurations $2 \ell 6 \ell$ '. A first one-electron transition, populating the $3 \ell$ orbital of $\mathrm{C}$, is followed by a correlated transfer excitation (CTE) process [22], involving a two-electron transition, into $2 \ell 6 \ell^{\prime}$. It is noted that both CDC and CTE processes, caused by the electron-electron interaction, are examples of the dielectronic processes of autoexcitation.

Concerning the configurations of quasi equivalent electrons $n \ell n^{\prime} \ell^{\prime}(n \approx n$ '), the situation is less clear, since they can be produced either by monoelectronic or by dielectronic processes [11,34]. For example, in the case of $\mathrm{N}^{7+}+$ He collisions, the mechanisms responsible for the capture into $3 \ell 3 \ell^{\prime}$ configurations depend onto the projectile velocity [11]. At $v_{p r o j} \sim 0.1$ a.u., these configurations are populated by two independent one-electron transitions. At higher impact velocities, a direct transition between the entrance channel and the final state is necessary to explain such population. For the system $\mathrm{C}^{6+}+\mathrm{He}$, it has been shown theoretically that the configurations of quasi equivalent electrons $2 \ell 3 \ell^{\prime}$ and $2 \ell 4 \ell^{\prime}$ are due to correlation effects [35] in a projectile velocity range from 0.6 a.u. down to 0.1 a.u. All these examples suggest that no general consideration can be made for quasi equivalent electron configurations. Our attention will be concentrated on non equivalent electron configurations, since interesting general results are found.

\section{Experimental set-up}

All the measurements reported here were carried out at the $14-\mathrm{GHz}$ electron cyclotron resonance (ECR) ion sources at the Grand Accélérateur National d'Ions Lourds (GANIL) in Caen and at the Ionenstrahl-Labor of the Hahn-Meitner Institut (HMI) in Berlin. The extracted ions were colliding an effusive gas jet. The Auger electrons produced after the collision were detected at angles ranging from 20 to $160^{\circ}$, with respect to the incident beam direction, using a single-stage spectrometer developed at HMI [36] which consists of an electrostatic parallel-plate analyzer. At the exit of the spectrometer, the Auger electrons were detected by using a channel electron multiplier.

Alternatively, as mentioned in the introduction, the RIMS was also used $[6,7,19]$. The incident beam was colliding a supersonic gas jet in order to have target atoms initially monocinetic. The scattered projectile ions were charged state analyzed and detected on a position sensitive detector (PSD). The recoil target ions were directed into a large diameter PSD. A strong electric field was needed to maintain a $4 \pi$ solid angle efficiency. From the time of flight (TOF) and the impact position of the collision partners, the momentum $\vec{p}_{r}$ of the recoil ions was determined. From the evaluation of $\vec{p}_{r}$, the projectile scattering angle $\theta$ as well as the inelasticity of the collision ( $Q$-value) were deduced [7]. These quantities were used to identify the populated configurations and the mechanisms responsible for the creation of these configurations. 


\section{Spectra analysis}

Figure 2 shows typical $K$-Auger spectra obtained at high resolution for the collision systems $\mathrm{C}^{6+}+\mathrm{He}$ (left side) and $\mathrm{N}^{7+}+\mathrm{He}$ (right side) [37], at projectile energies in the range from $3.5 \mathrm{keV}$ to $132 \mathrm{keV}$. The observation angle is $40^{\circ}$ with respect to the incident beam direction. The peaks are attributed to the configurations $2 \ell \mathrm{n} \ell^{\prime}(\mathrm{n} \geq 3)$, which decay to the $2 \ell \varepsilon \ell^{\prime}$ continuum by means of Auger transitions. Auger electrons from the configurations $3 \ell n \ell^{\prime}(n \geq 3)$ have been also observed in the present experiments. The associated peaks due to $M$-Auger transitions are observed at electron energies lower than $80 \mathrm{eV}$.
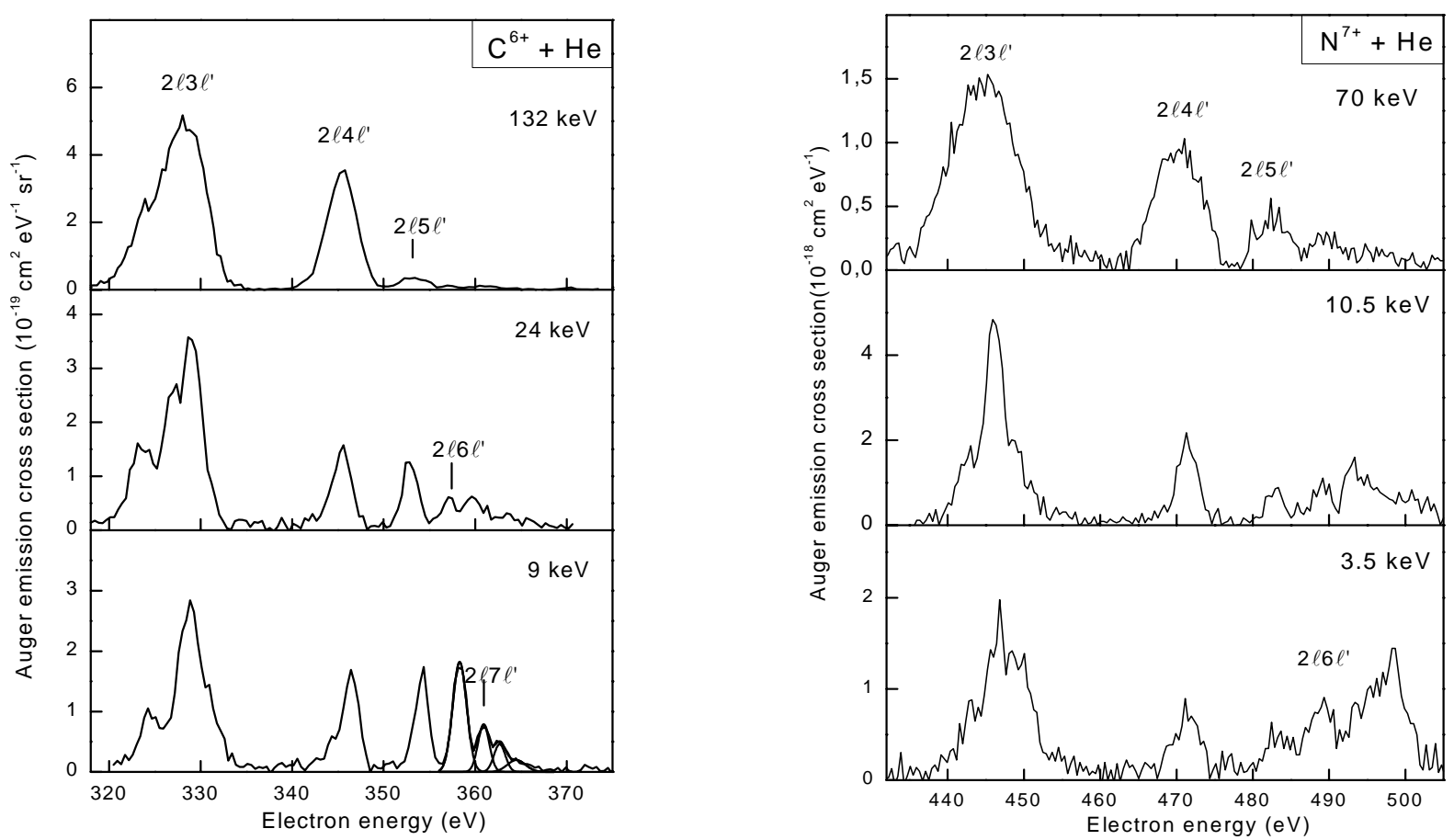

Figure 2. High-resolution spectra of $K$-Auger electrons produced in $\mathrm{C}^{6+}+\mathrm{He}$ and $\mathrm{N}^{7+}+\mathrm{He}$ collisions at a few projectile energies.

Figure 2 indicates that, at the highest projectile energy, Auger electron emission originates primarily from quasi equivalent electron configurations $2 \ell 3 \ell^{\prime}$ and $2 \ell 4 \ell^{\prime}$. Configurations of non equivalent electrons are also visible but represent less than $10 \%$ of the total intensity. In contrast, at lower projectile energies, the production of non equivalent electron configurations increases strongly. The increase of the population of such configurations is significant for $\mathrm{C}^{6+}+\mathrm{He}$ collisions. At collision energies lower than $10 \mathrm{keV}$, Auger intensities due to the configurations $2 \ell 5 \ell^{\prime}$ and $2 \ell 6 \ell^{\prime}$ is as large as that of the peaks originating from $2 \ell 4 \ell$ '. Furthermore, configurations $2 \ell$ n $\ell^{\prime}$ involving principal quantum numbers $\mathrm{n}$ as high as 9 are clearly visible.

For the system $\mathrm{N}^{7+}+\mathrm{He}$, the increase is also visible, but is less pronounced than that for the system $\mathrm{C}^{6+}+$ He. The configurations $2 \ell \mathrm{n} \ell$ ', with $\mathrm{n}=3-5$ remain constant in the whole range of projectile energies. The intensity due to the configurations involving $\mathrm{n} \geq 6$ increases by a factor of $\sim 10$ when the projectile energy decreases from $70 \mathrm{keV}$ to $3.5 \mathrm{keV}$. 
Average Auger yields and double capture cross sections

For all the collision systems studied here, the Auger spectra were used to determine the corresponding cross sections. First, the Auger spectra were integrated to obtain single differential cross sections $d \sigma_{n n^{\prime}}^{a} / d \Omega$ for Auger-electron emission attributed to a given configuration $\mathrm{n} \ell \mathrm{n}$ ' $\ell$ '. The total cross sections $\sigma_{n n^{\prime}}^{a}$ were then deduced by integration of $d \sigma_{n n^{\prime}}^{a} / d \Omega$ over the electron-emission angle. To determine total double-capture cross sections $\sigma_{n n^{\prime}}$, the quantity $\sigma_{n n^{\prime}}^{a}$ was divided by the corresponding average Auger yield $a_{n n}$ '. This Auger-yield correction is due to the fact that configurations $\mathrm{n} \ell$ ' $\ell$ ' may also decay via radiative transitions which compete with the Auger transitions.

Auger yields integrated over $\ell$ and $\ell$ ' could be directly deduced from RIMS for the collisions $\mathrm{C}^{6+}+\mathrm{He}$ and $\mathrm{Ne}^{10+}+\mathrm{He}[19,33]$. Double differential cross sections (DDCS) for autoionizing (ADC) and radiative (RDC) double capture were extracted from the experiment as a function of the energy gain $Q$ and the projectile scattering angle $\theta$. The DDCS were integrated over $\theta$. Results are shown in Figure 3 for ADC and RDC following $\mathrm{C}^{6+}+\mathrm{He}$ collisions at projectile energies of 9, 30 and $90 \mathrm{keV}$. The results for $a_{n n}$, are reported in Table 1. It is seen that the Auger yields are rather insensitive to the projectile energy, within the uncertainties. This finding is in different from previous conclusions, which were supported by experiments [38]. In these experiments, the stabilization ration $R$, defined by $R=1-a$, where $a$ is the Auger yield averaged on all the configurations, was presented for various systems. The authors found an increase of $R$ with decreasing the projectile velocity. As an explanation, they suggested that this can be due to an increase of individual stabilization ratio $R_{n n}$ ' (i.e. $1-a_{n n}$ ) caused by a modification of the populated $2 \ell \mathbf{n} \ell^{\prime}$ configurations in the electric field of the recoil ion leading to a shift to high- $\ell$ values. [38]. Rather, the increase of $R$ is due to the increase of cross sections for populating configurations of non equivalent electrons $2 \ell \mathrm{n} \ell^{\prime}(\mathrm{n} \geq 4)$.

Table 1. Experimental and theoretical Auger yield $a_{n n}$, associate with the configurations $2 \ell \mathbf{n} \ell^{\prime}$ of $C^{4+}$.

\begin{tabular}{c|ccc|c}
\hline \hline \multirow{2}{*}{$2 \ell 3 \ell^{\prime}}$, & \multicolumn{3}{|c|}{ Experiment [33] } & Calculation [22] \\
\cline { 2 - 5 } $2 \ell 4 \ell^{\prime}$ & $9 \mathrm{keV}$ & $30 \mathrm{keV}$ & $90 \mathrm{keV}$ & $60 \mathrm{keV}$ \\
\hline $2 \ell 5 \ell^{\prime}$ & $0.77 \pm 0.04$ & $0.86 \pm 0.02$ & $0.81 \pm 0.01$ & 0.66 \\
$2 \ell 6 \ell^{\prime}$ & $0.26 \pm 0.03$ & $0.37 \pm 0.12$ & $0.44 \pm 0.15$ & 0.53 \\
$2 \ell 7 \ell^{\prime}$ & $0.15 \pm 0.02$ & $0.19 \pm 0.11$ & - & 0.35 \\
$2 \ell 8 \ell^{\prime}$ & $0.13 \pm 0.04$ & $0.11 \pm 0.12$ & & 0.29 \\
$2 \ell 9 \ell^{\prime}$ & & & - & 0.18 \\
& & & - & 0.15 \\
\hline \hline
\end{tabular}




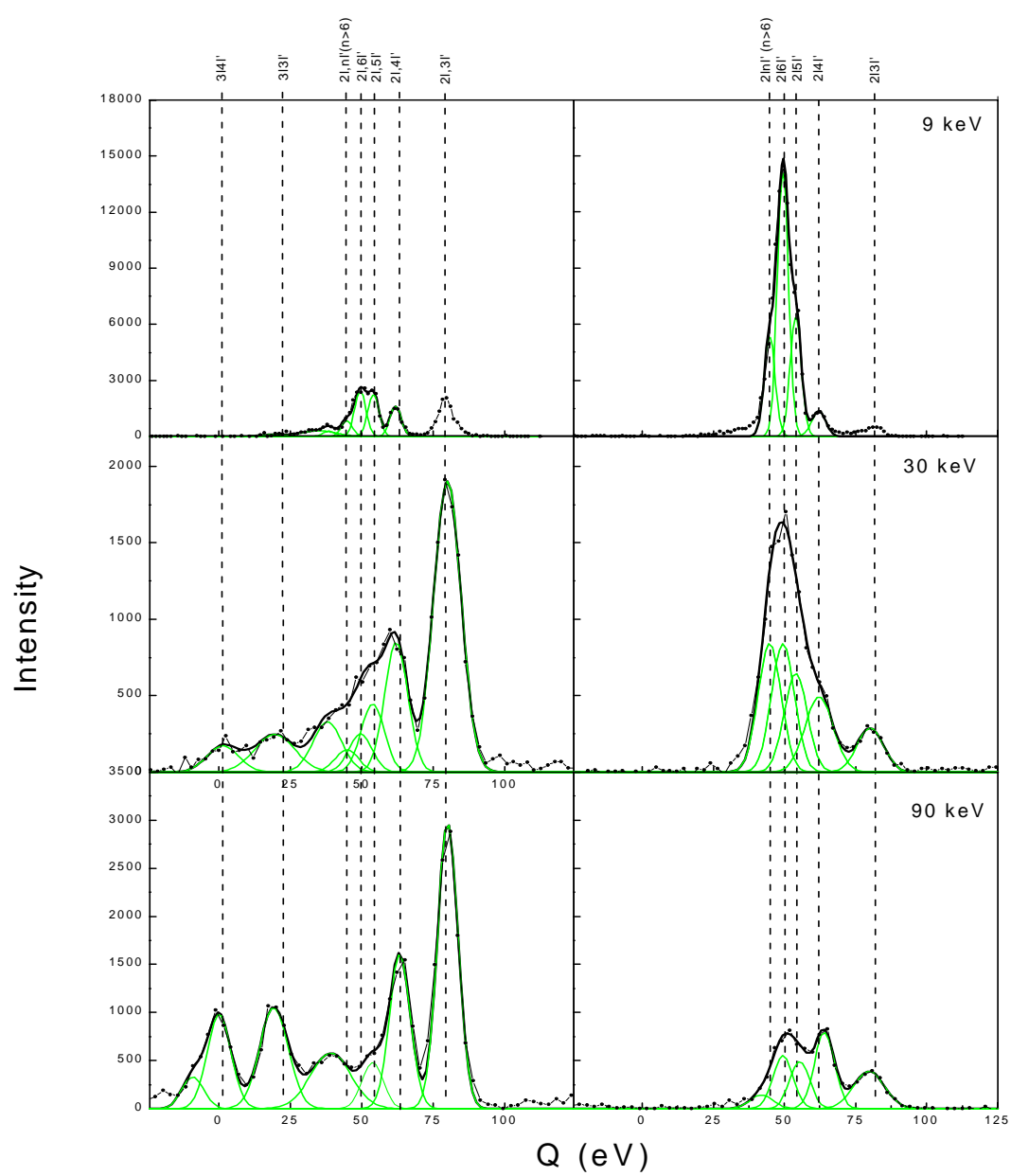

Figure 3. Experimental Q-value distributions for autoionizing (left side) and radiative (right side) DC in $\mathrm{C}^{6+}+$ He collisions.

The experimental Auger yields are also compared (right side of Table 1) with theoretical calculations. The calculated Auger yields $a_{n n}$, were obtained by means of the Hartree-Fock code of Cowan [39]. Since the quantity $a_{n n}$ ' depends strongly on the occupation probabilities associated with the quantum numbers $\ell$ and $\ell$, various distributions were tested [40]. Moreover, calculations have been done considering the variation of these probabilities with respect to the collision energy. As a result, it was found that $a_{n n}$ ' are rather constant for all the collision energies, which is consistent with the experiment.

Double capture cross sections (DCCS) $\sigma_{n n^{\prime}}$ for the system $\mathrm{C}^{6+}+\mathrm{He}$ are shown in Figure 4 as a function of the impact velocity. The quantity $\sigma_{n n^{\prime}}$ deduced from Auger electron measurements is compared with the experimental cross sections extracted from RIMS [33] and with theoretical calculations $[11,15]$. The framework of the theoretical method is the same as that described in Refs. $[35,41]$. In a straight-line impact-parameter treatment, the total electronic wave function is expanded onto a set of configurations built with product of one-electron diatomic-molecule (OEDM) orbitals, which are exact solutions of one-electronic two-center Hamiltonian. The introduction of this expansion in the Schrödinger equation leads to a set of coupled equations that is numerically integrated [42]. First, a good overall agreement is seen for all the configurations. The experiments and the theory reproduce quite well the same variation of $\sigma_{n n^{\prime}}$ with the projectile velocity. A noticeable disagreement is seen for the configurations $2 \ell 6 \ell^{\prime}$ at the lowest velocities. This is partially due to the limitation of the theory which represents configurations such as $2 \ell 6 \ell^{\prime}$ ' with only a few states [19]. 


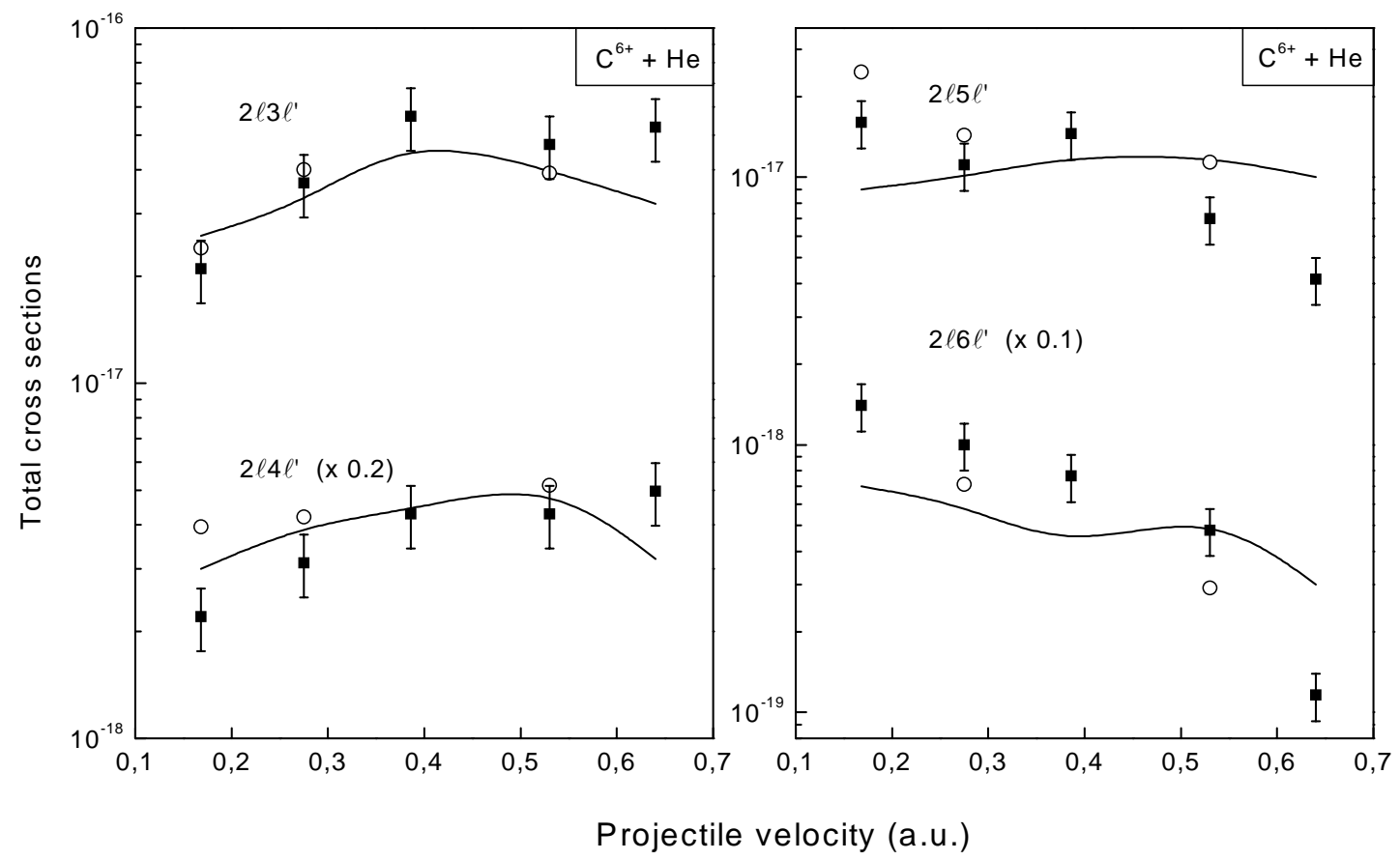

Figure 4. Total cross sections for the production of the configurations $2 \ell n \ell^{\prime}(n=3-7)$ in $C^{6+}+H e$ collisions. Experimental results are given by full squares [37] and open circles [33]. Solid lines show the theoretical results [15]

The variations of the cross sections with the projectile velocity were also analyzed in the case of $\mathrm{N}^{7+}$ and $\mathrm{O}^{8+}$ projectiles. The DCCS for populating the configurations $2 \ell \mathrm{n} \ell^{\prime}$ and $3 \ell \mathrm{n} \ell^{\prime}$ are presented in Figure 5 , for $\mathrm{N}^{7+}+\mathrm{He}$ (left side) and $\mathrm{O}^{8+}+\mathrm{He}$ (right side) collisions, respectively. While at high velocities the configurations of quasi equivalent electrons are mainly populated, the configurations of non equivalent electrons gain importance since the projectile velocity decreases. At the lowest velocities, they are found to be dominant in the case of $\mathrm{N}^{7+}+$ He collisions.

The ratio $R_{N E}$ between DCCS associated with non equivalent electron configurations and total DCCS is summarized in Table 2 for both the lowest and highest velocitiesstudied here, i.e. 0.01 a.u. and 0.6 a.u., respectively. The strong increase with decreasing the projectile velocity is confirmed for all the configurations, except for the series $3 \ell \mathrm{n} \ell^{\prime}$ populated in $\mathrm{N}^{7+}+\mathrm{He}$ collisions. Note that the configurations $3 \ell \mathrm{n} \ell^{\prime}$ (resp. $4 \ell \mathrm{n} \ell^{\prime}$ ) for $\mathrm{C}^{6+}+\mathrm{He}$ (resp. $\mathrm{Ne}^{10+}+\mathrm{He}$ ) collisions are not reported in Table 2 , since they are missing in our experiments.

Table 2. Contribution of non equivalent electron configuration to total double electron capture.

\begin{tabular}{c|c|cc}
\hline \hline Projectile & Configuration & \multicolumn{2}{|c}{ Ratio $R_{N E}$} \\
\hline \multirow{3}{*}{$\mathrm{C}^{6+}$} & & $V_{\text {proj }} \sim 0.6$ a.u. & $V_{\text {proj }} \sim 0.01$ a.u. \\
\cline { 3 - 4 } $\mathrm{N}^{7+}$ & $2 \ell \mathrm{n} \ell^{\prime}(\mathrm{n} \geq 4)$ & 0.16 & 0.81 \\
$\mathrm{~N}^{7+}$ & $2 \ell \mathrm{n} \ell^{\prime}(\mathrm{n} \geq 4)$ & 0.10 & 0.68 \\
$\mathrm{O}^{8+}$ & $3 \ell \mathrm{n} \ell^{\prime}(\mathrm{n} \geq 5)$ & 0.026 & 0.043 \\
$\mathrm{Ne}^{10+}$ & $3 \ell \mathrm{n} \ell^{\prime}(\mathrm{n} \geq 5)$ & 0.04 & 0.17 \\
\hline \hline
\end{tabular}



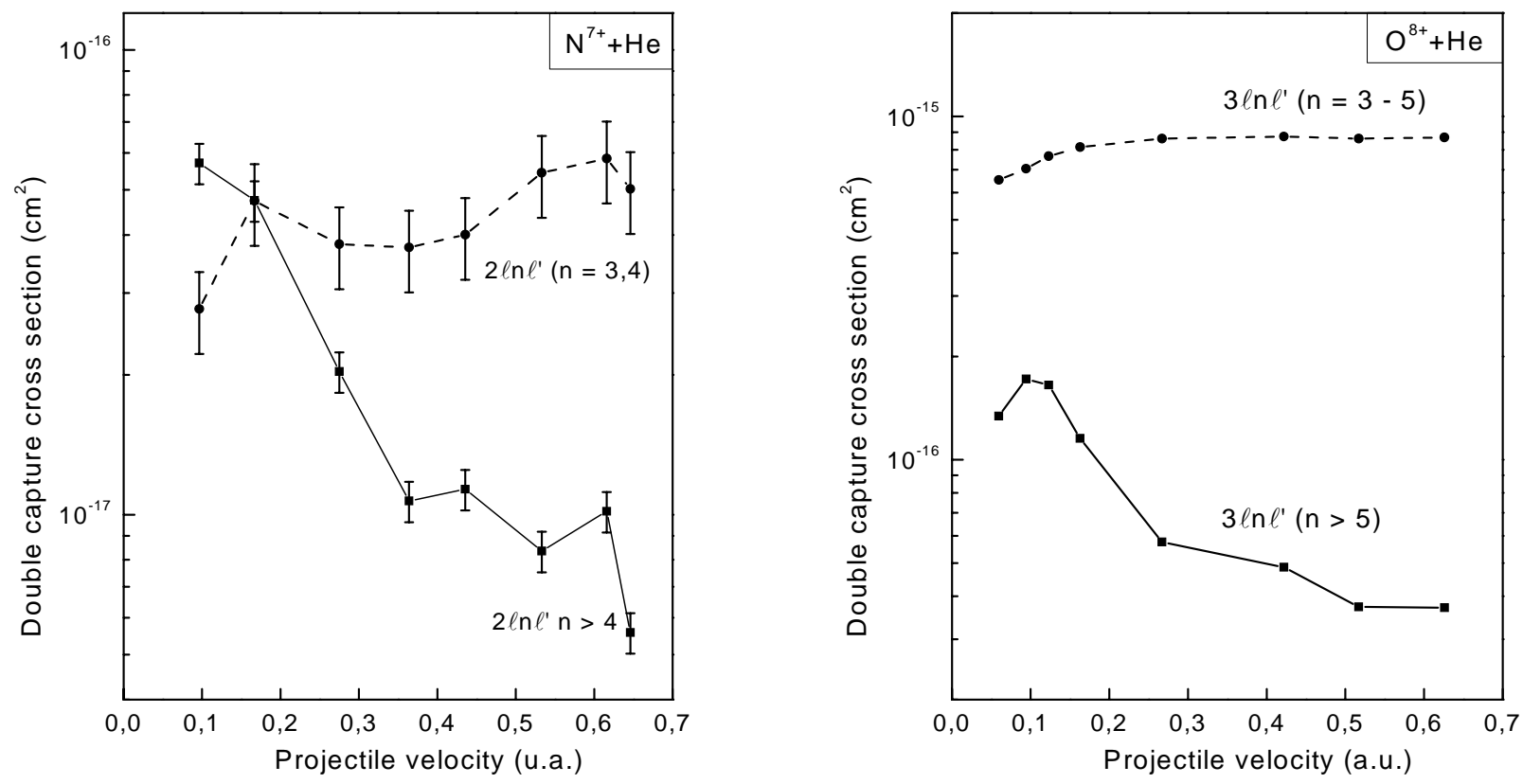

Figure 5. DC cross section for populating configurations of quasi equivalent and non equivalent electrons for $\mathrm{N}^{7+}+\mathrm{He}$ and $\mathrm{O}^{8+}+\mathrm{He}$ collisions as a function of the projectile velocity.

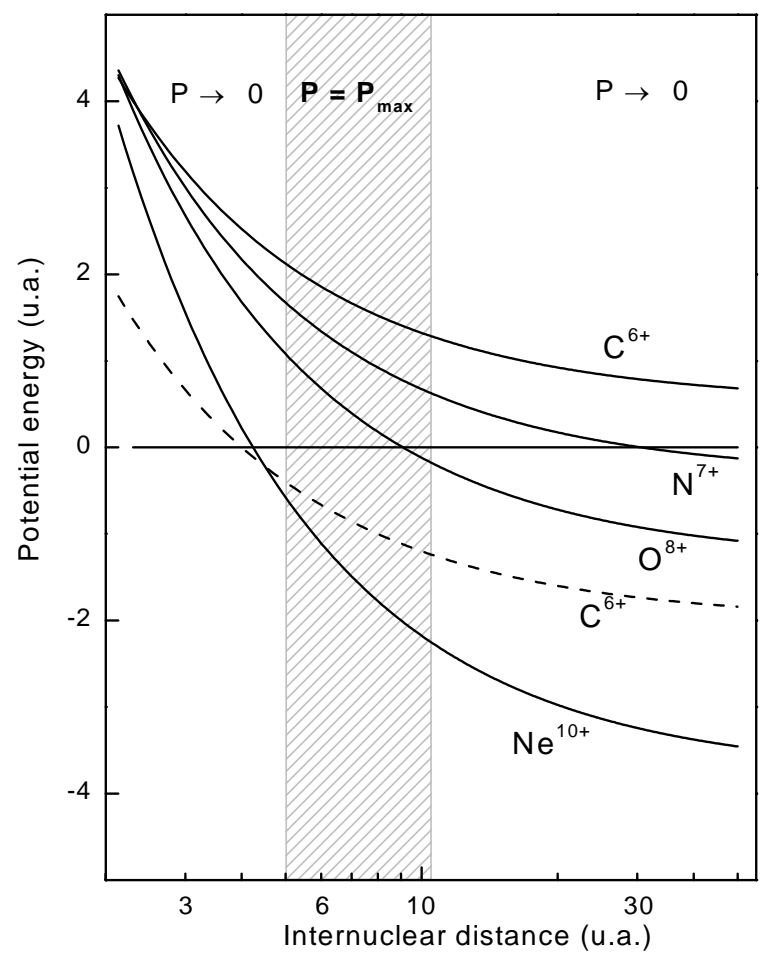

Figure 6. Schematic diagram of potential energies for the systems $A^{Z+}+H e(Z=6,7,8$ and 10) in the case of the configurations $3 \ell 6 \ell^{\prime}$ (solid curves) and $2 \ell 6 \ell^{\prime}$ (dashed curve). 
As seen in Table 2, the systematic study of $A^{Z+}+$ He collisions $(Z=6-10)$ shows clearly the important role of electron correlation effects, leading to non equivalent electron configurations, especially at very low impact velocities. Moreover, independent models such as COB model which predict fairly well the main quantities (principal quantum numbers, total cross sections) at impact velocities of the order of 0.5 a.u. fail to reproduce the experiment at low projectile velocity. Hence, DC process cannot be treated anymore using independent transitions. In other words, the collision is a true many-body problem at low impact velocities.

Table 2 also shows that, at $v_{\text {proj }} \sim 0.01$ a.u., the contribution of dielectronic processes to total DC capture depends on the projectile charge. For example, whereas $\sim 60 \%$ of the configurations $3 \ell$ n $\ell^{\prime}$ $(\mathrm{n} \geq 5)$ contribute to the total DC in $\mathrm{Ne}^{10+}+\mathrm{He}$ collisions, the population of these configurations following $\mathrm{N}^{7+}+\mathrm{He}$ collisions remains negligible. This finding has been already explained with the help of the crossing radius $R_{c}$ between the entrance channel and the DC channel (CDC mechanism) [24]. The quantities $R_{c}$ are visualized in Figure 6 for the configurations $3 \ell 6 \ell$ ' following the impact of $\mathrm{A}^{\mathrm{Z}+}$ ions, where $Z$ is the atomic number. The dashed region represents the internuclear distances at which the double capture probability, evaluated with the Landau-Zener model, is maximum. It is seen that the crossing radius increases with decreasing the projectile charge. Hence, while transitions into $3 \ell 6 \ell$ ' configurations are favored in $\mathrm{O}^{8+}+\mathrm{He}$ and $\mathrm{Ne}^{10+}+\mathrm{He}$ collisions, the DC probability is negligible for $Z=6$ and 7 , due to the strong diabaticity of the crossings. This expectation is consistent with the contributions of $3 \ell \mathbf{n} \ell^{\prime}(n>5)$ deduced from the experiments (Table 2$)$. In addition, the configuration $2 \ell 6 \ell^{\prime}$ has been represented in Figure 6 for the collision $\mathrm{C}^{6+}+$ He. The crossing leads also to a large transition probability. Hence, the configurations $2 \ell \mathbf{n} \ell^{\prime}$ and $3 \ell \mathbf{n} \ell^{\prime}$ play the same role in the collisions $\mathrm{C}^{6+}+\mathrm{He}$ and $\mathrm{Ne}^{10+}+\mathrm{He}$, respectively. It would be interesting to investigate higher charged systems such as $\mathrm{Ar}^{14+}+\mathrm{He}$, for which the configurations $4 \ell \mathrm{n} \ell$ ' with $\mathrm{n}>6$ are expected to play a decisive role [43].

\section{Identification of monoelectronic and dielectronic processes}

To identify more precisely the mechanisms responsible for the population of non equivalent electron configurations, close-coupling calculations of DDCS using the method described above were performed and compared with experimental distributions [19,33]. Both distributions are shown in Figure 7 for the series $3 \ell \mathrm{n} \ell^{\prime}$ and $4 \ell \mathrm{n} \ell^{\prime}(\mathrm{n} \geq 4)$ populated during the collision $\mathrm{Ne}^{10+}+\mathrm{He}$ at an energy of $150 \mathrm{keV}$. The agreement is rather good, especially for the configurations $4 \ell \mathbf{n} \ell$ '. Discrepancies appear for configurations $3 \ell 4 \ell^{\prime}$ and $3 \ell 7 \ell^{\prime}$ in the shape as well as in the intensity of the distributions. Because of the good accuracy in the calculations, test calculations were performed for the configurations $4 \ell 4 \ell^{\prime}$ and $3 \ell 6 \ell^{\prime}\left(\mathrm{Ne}^{10+}+\mathrm{He}\right)$ and the configurations $2 \ell \mathrm{n} \ell^{\prime}\left(\mathrm{C}^{6+}+\mathrm{He}\right)$, in which dynamic couplings between the entrance channel and several capture channels were cancelled. These test calculations were useful to reveal the importance of the electron-electron interaction [19].

In parallel with the close-coupling test calculations [19], the mechanisms could be partially separated by analyzing the different capture pathways leading to the population of a given configuration [33]. As an example, the configuration $3 \ell 6 \ell^{\prime}$ may be populated

- by two monoelectronic transitions due to electron-nucleus (EN) interaction via the intermediate channel $\mathrm{Ne}^{9+}+\mathrm{He}^{+}$;

- by a CDC process (direct transition);

- by a CTE process involving the intermediate $4 \ell$ or $5 \ell$ orbitals of $\mathrm{Ne}^{9+}$. 


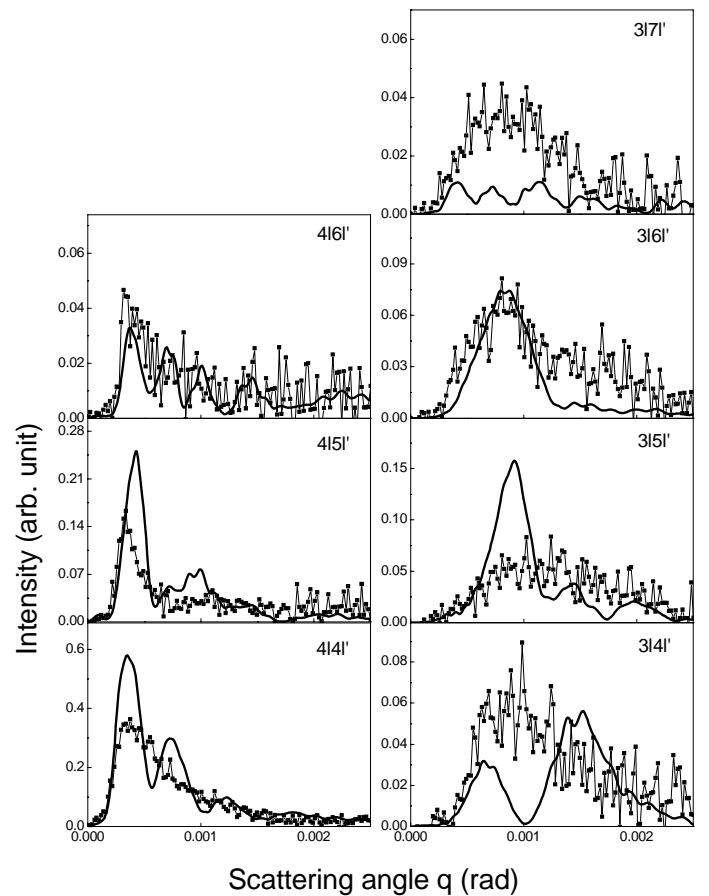

Figure 7. Relative differential DC cross sections in $\mathrm{Ne}^{10+}+\mathrm{He}$ collisions. Full curves, theory [19]; squares, experiment.

These three pathways correspond to classical projectile scattering angles $\theta_{\text {proj }}$ that are well separated from each other. The detailed analysis reported in Ref. [33] leads to the identification of the possible mechanisms. The results are reported in Table 3 for the collision systems $\mathrm{C}^{6+}+\mathrm{He}$ and $\mathrm{Ne}^{10+}+\mathrm{He}$. First, it is seen that both close-coupling and classical-trajectory calculations predict similar mechanisms. As expected, the configurations of non equivalent electrons are shown to be populated by dielectronic processes. In addition, the configurations of quasi equivalent electrons $2 \ell 3 \ell^{\prime}\left(\mathrm{C}^{6+}+\mathrm{He}\right)$ are also populated by dielectronic processes involving a direct transition with the entrance channel $(C D C)$. The classical calculations predict that, in $\mathrm{Ne}^{10+}+\mathrm{He}$ collisions the $3 \ell 4 \ell$ ' configurations are populated by CDC processes, while $4 \ell 5 \ell^{\prime}$ and $4 \ell 6 \ell^{\prime}$ may be created by either electron-nucleus (EN) or electron-electron (CDC or CTE) interaction. More theoretical tests are needed to confirm these predictions. However, both calculations confirm that dielectronic processes play a major role in ion atom collisions (see Table 3).

Table 3. Mechanisms responsible for DC in $\mathrm{C}^{6+}+\mathrm{He}$ and $\mathrm{Ne}^{10+}+\mathrm{He}$ collisions

\begin{tabular}{c|cccc}
\hline \hline & \multicolumn{2}{|c}{$\mathrm{C}^{6+}+\mathrm{He}$} & \multicolumn{2}{c}{$\mathrm{Ne}^{10+}+\mathrm{He}$} \\
Configurations & OEDM [19] & Classical [33] & OEDM [19] & Classical [33] \\
\hline $3 \ell 3 \ell^{\prime}$ & $\mathrm{EN}$ & $\mathrm{EN}$ & - & - \\
$3 \ell 4 \ell^{\prime}$ & - & - & - & $\mathrm{CDC}$ \\
$3 \ell \mathrm{n} \ell^{\prime} \mathrm{n} \geq 5$ & - & - & $\mathrm{CTE}(4 \ell)$ & $\mathrm{CTE}(5 \ell)$ \\
$4 \ell 4 \ell^{\prime}$ & - & - & $\mathrm{EN}$ & EN or CDC \\
$4 \ell 5 \ell^{\prime}$ & - & - & - & EN or CDC \\
$4 \ell 6 \ell^{\prime}$ & - & - & - & EN or CTE (5 $)$ \\
$2 \ell 3 \ell^{\prime}$ & $\mathrm{CDC}$ & $\mathrm{CDC}$ & - & - \\
$2 \ell \mathrm{n} \ell^{\prime} \mathrm{n} \geq 4$ & $\mathrm{CTE}(3 \ell)$ & $\mathrm{CTE}(3 \ell)$ & - & - \\
\hline \hline
\end{tabular}




\section{Ion-molecule collisions - Dynamics of the molecular fragmentation}

\section{Presentation}

As mentioned briefly in the introduction, the knowledge of molecular fragmentation dynamics is one of the main goal in the study of slow ion-molecule collisions. In particular, much work has been devoted to simple molecular targets such as $\mathrm{D}_{2}$ and $\mathrm{H}_{2}$ [27,31,44] or $\mathrm{CO}$ [28,45-47],. However, the fragmentation depends strongly onto the primary process, i.e. the electron capture, which is responsible for the ejection of target electrons. Hence, the fragmentation dynamics is complicated for a multi electron target. For this reason, our discussion will be focused on the analysis of the $\mathrm{H}_{2}$ molecular target fragmentation following the impact of highly charged projectiles.

At projectile velocities $v_{\text {proj }}$ larger than 0.1 a.u., the collision time is of the order of $10^{-15} \mathrm{~s}$, which is much shorter than the characteristic times in the molecule $\left(\sim 10^{-13} \mathrm{~s}\right)$. Thus, the collision can be divided into two independent steps [25]. The first step is the collision itself. During the collision, one or two electron from $\mathrm{H}_{2}$ may be captured :

$$
A^{q+}+H_{2} \rightarrow A^{(q-1)+}(n \ell)+H_{2}^{+}
$$

for a SC process, and

$$
A^{q+}+H_{2} \rightarrow A^{(q-2)+}\left(n \ell n^{\prime} \ell^{\prime}\right)+H_{2}^{2+}
$$

for a DC event.

The second step is the fragmentation of the residual ionized target. It has been shown previously that, at velocities of $\sim 0.5$ a.u., SC leads to an ionized target $H_{2}^{+}$which is mainly in its fundamental state, so that no dissociation of the target is expected. Hence, the kinetic energy of the $H_{2}^{+}$ion originates only from the recoil energy $E_{r}$ transferred from the projectile to the center of mass of the target during the collision. In contrast, DC leads to $\mathrm{H}_{2}^{2+}$ which dissociates, giving rise to protons whose kinetic energy $E_{f}$ in the framework of the molecular center of mass is of the order of $9.5 \mathrm{eV}$ if they make a vertical Franck-Condon transition from the molecular ground state.

It has been shown recently [31,48,49] that the final energy of a fragment following dissociation after DC process has two sources : the recoil energy $E_{r}$ transferred by the projectile to the center of mass of the ionized target, and the fragment energy $E_{f}$ originating from the Coulomb dissociation. However, it has been recognized very recently that this two-step picture is too simple since the fragments may also be influenced by the projectile $[47,44]$. The interaction between the projectile and one fragment after the capture (also denoted post collision interaction) has been evidenced experimentally in the fragmentation of $\mathrm{D}_{2}$ following the impact of highly charged $\mathrm{Xe} \mathrm{e}^{23+}$ projectiles, at impact energies of 0.2 and $0.8 \mathrm{keV} / \mathrm{u}$ [44]. In the CM of the residual $D_{2}^{2+}$ ion, the maximum of the fragment kinetic energy distribution was found to be shifted to energies smaller than $9.5 \mathrm{eV}$. In addition, an increase in the width of the distribution was observed when decreasing $v_{\text {proj. }}$ This is due to the fact that, since $v_{\text {proj }}$ decreases, there is more time for the fragments to separate from their initial positions while the projectile field is still present.

In order to analyze the influence of the projectile, experiments were performed at energies ranging from $1.25 \mathrm{keV}$ ( $v_{p r o j}=0.055$ a.u.) up to $105 \mathrm{keV}$ ( $v_{p r o j}=0.51$ a.u.). Figure 8 shows ion spectra for the system $\mathrm{O}^{5+}+\mathrm{H}_{2}$ at projectile energies of 2.5 and $75 \mathrm{keV}$, and for detection angles of $20^{\circ}$ and $90^{\circ}$. At these energies, the peaks are found to be located around $9.5 \mathrm{eV}$, as indicated by the arrows on Figure 8 . Nevertheless, a closer inspection of the spectra shows that the mean energy of a fragment increases for increasing the detection angle. This shift is seen to be more pronounced when $v_{\text {proj }}$ decreases. Moreover, a double structure is observed at the lowest energy. 
To interpret the energy shift of the observed distributions, which is expected to originate from the Coulomb repulsion of the projectile nucleus, we performed calculations using two simple classical models. Since these models have been described previously in details [31,47], only a brief description is given here.

In the first model, referred to as two-step two-body (TS2B) model, the problem was treated as two successive two-body problems. First the ionized target recoils with a velocity $\vec{v}_{r}$. Then, the ion dissociates with a velocity $\overrightarrow{\boldsymbol{V}}_{f}^{C M}$ in the molecular CM frame. Thus, the detected proton has a velocity $\overrightarrow{\boldsymbol{V}}_{f}^{L}=\overrightarrow{\boldsymbol{V}}_{f}^{C M}+\overrightarrow{\boldsymbol{V}}_{r}$ in the laboratory frame. To evaluate $\overrightarrow{\boldsymbol{V}}_{r}$, expressions for the longitudinal $p_{/ /}$and transverse $p_{\perp}$ momenta of the recoiling target, which depend onto the inelastic energy transfer $Q$ and the initial projectile momentum $p_{o}$, were calculated. From $p_{/ /}$and $p_{\perp}$, the recoil energy $E_{r}$ and the recoil angle $\psi_{r}$ were deduced and utilized to determine the average energy of the distributions as a function of the detection angle.

In the second model, classical kinematics equations were used. In this model, a sudden double capture was first assumed, at an impact parameter of about 5 a.u., which is the value expected for the maximum of DC probability. In addition DC was assumed to occur in a plane perpendicular to the initial velocity of the projectile and that contains the molecule CM. Hence, the problem to solve is a pure three-body problem involving Coulomb interactions between the three collision partners. A set of nine coupled equations was solved numerically and the energy of each proton was deduced. Note that in contrast with the TS2B model, the second model, referred to as one-step three-body (OS3B) model, takes into account the post collision interaction.

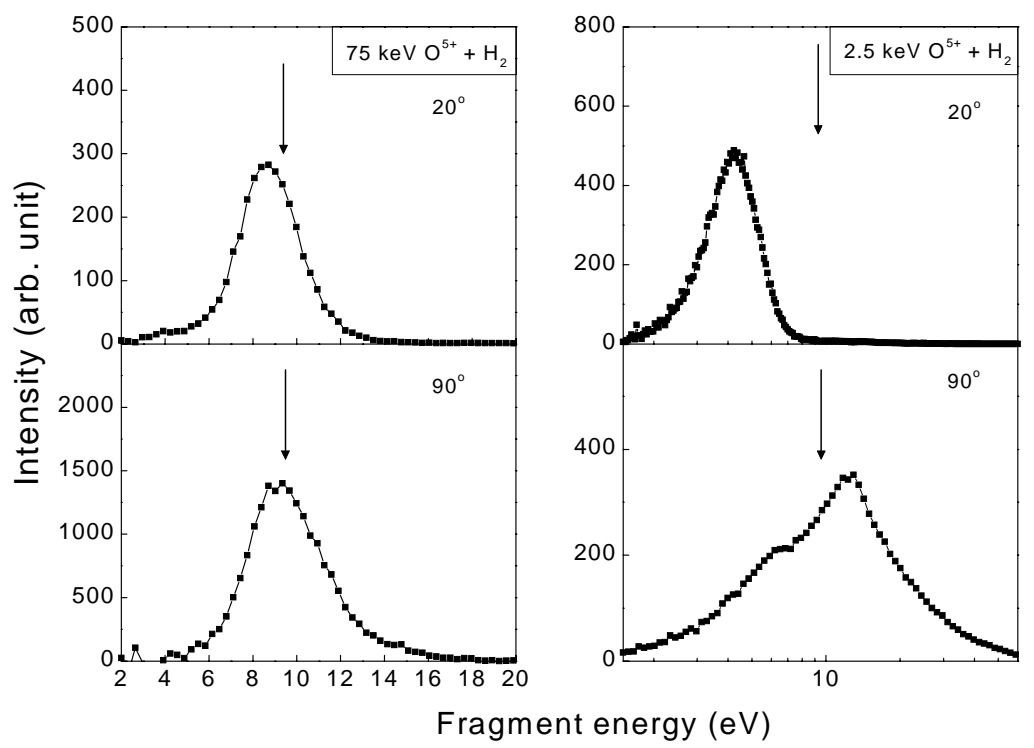

Figure 8. Energy distributions of the protons at detection angles of $20^{\circ}$ and $90^{\circ}$ following the fragmentation of $\mathrm{H}_{2}^{2+}$ ions after DC in $75 \mathrm{keV}$ and $2.5 \mathrm{keV} \mathrm{O}{ }^{5+}+\mathrm{H}_{2}$ collisions. The arrow indicates the expected energy assuming a free fragmentation.

\section{Energy distributions and cross sections}

A comparison between the experimental energy maxima (full squares) and the calculation (dashed curves and solid curves for the TS2B and OS3B models, respectively) is presented in Figure 9 for two 
projectile energies $(75 \mathrm{keV}$ and $2.5 \mathrm{keV})$. The experimental values were determined by fitting the spectra with Gaussian curves. At the highest projectile energy, both models agree well with experimental data. In other words, the PCI is found to be negligible, as suggested previously [31, 47,44]. The small energy shift observed in Figure 9 is only due to kinematic rules. At $75 \mathrm{keV}$, the mean energy of the recoiling $\mathrm{CM}$ is of the order of $0.05 \mathrm{eV}$, which leads to a mean velocity $v_{r}$ of about $10^{-3}$ a.u. Since the velocity of a fragment in the case of a pure fragmentation is $\sim 0.0195$ a.u., the total velocity of a fragment is in the range $0.0185-0.0205$ a.u., corresponding to energies in the laboratory frame ranging from $8.55 \mathrm{eV}$ to $10.5 \mathrm{eV}$. These values are consistent with the minimum and the maximum of experimental energies (Figure 9).

In contrast, at the lowest energy, differences between the models and experiment appear (right side of Figure 9). While both models reproduce qualitatively the double structure observed, quantitative discrepancies occur, especially at forward detection angles. At these angles, the agreement between experiment and the model calculation is found to be better when using the OS3B model. In other words, this indicates that the post-collision interaction plays a role. However, the results shown in Figure 9 depend strongly onto the initial conditions, so that the comparison between both models is questionable.
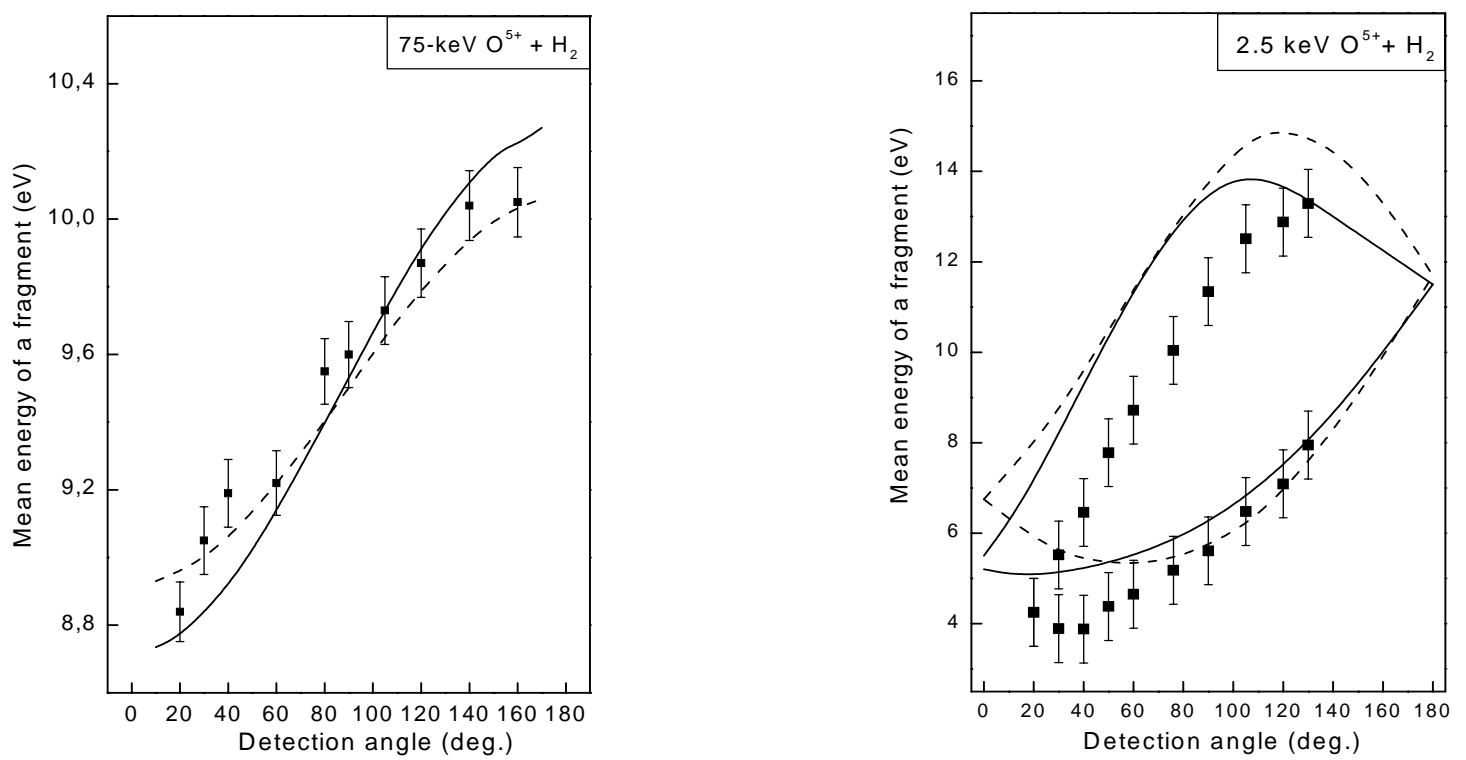

Figure 9. Maximum of fragment energy distribution following $\mathrm{O}^{5+}+\mathrm{H}_{2}$ collisions at $75 \mathrm{keV}$ and $2.5 \mathrm{keV}$. Full squares, experimental values; dashes curves, TS2B calculations; solid curves, OS3B calculations.

From model calculations, differential cross sections $d \sigma / d \theta$ for detecting one proton were also deduced, as a function of the detection angle. The results are reported in Figure 10 for two extreme projectile energies (10 keV and $1.25 \mathrm{keV}$ ). At the highest energy (left side of Figure 10), both models reproduce the experimental cross sections, which were determined by integration of the spectra over the fragment energy. Thus the influence of the projectile is negligible at this velocity. Moreover, it is seen that the cross section increases with increasing the detection angle. This is due to the fact that the recoil angle of the molecular $\mathrm{CM}$ moves towards angles larger than $90^{\circ}$. In other terms, the increase is only due to kinematic rules. 
In contrast, at $1.25 \mathrm{keV}$, large discrepancies appear between the models and the experiment. It is first seen that the increase of the results originating from the TS2B model is less pronounced than that from the OS3B model. This latter model is found to give the best agreement with the experiment. The present result shows again that the post-collision interaction has to be taken into account when the projectile velocity is sufficiently small.
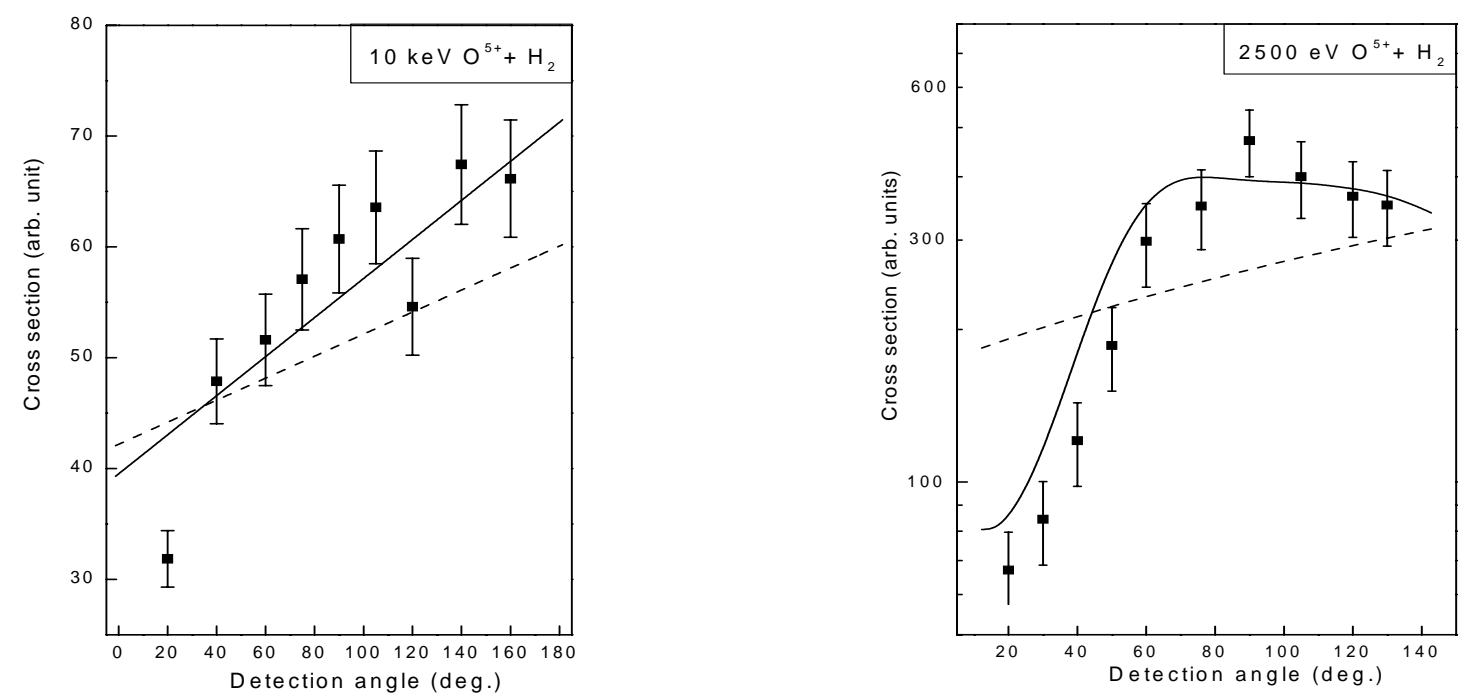

Figure 10. Differential cross section for detecting one fragment in $75 \mathrm{keV}$ and $2.5 \mathrm{keV} \mathrm{O}{ }^{5+}+\mathrm{H}_{2}$ collisions. Full squares, experimental values; dashes curves, TS2B calculations; solid curves, OS3B calculations.

\section{Conclusion}

To summarize, we studied the role of the projectile velocity in ion-atom and ion-molecule collisions. In ion atom collision, we emphasized one- and two-electron processes that occur during the collision. In particular, the electron-electron interaction was analyzed in $A^{Z+}+H e(Z=6-10)$ collisions. While the electron-nucleus interaction populates configurations of quasi equivalent electrons, the electron- electron interaction contributes dominantly to configurations of non equivalent electrons. When the velocity decreases, it is found that the contribution of the dielectronic processes due to electron correlation effects increases strongly. Hence, the picture of two independent transitions is no more valid to explain the double capture process at low impact velocity.

In the case of ion-molecule collisions, the velocity dependence reveals also two different regimes. At relatively large projectile velocities $(\sim 0.5$ a.u. $)$, for the system $\mathrm{O}^{5+}+\mathrm{H}_{2}$, the collision can be divided into two independent steps, including the collision and then the fragmentation of the residual ionized target. Hence, the problem to solve can be treated as two independent two-body problems. To give evidence for the specific role of the projectile, experimental measurements were performed at very low impact velocities ( $<0.1$ a.u.). The analysis of the fragment emission cross sections reveals that the protons are repulsed in backward directions, due to the Coulomb forces induced by the projectile. Similarly to the case of ion-atom collisions, at low velocities the problem to solve is a pure many-body problem, involving the three nuclei. 


\section{References and Notes}

1. Janev, R. K.; Winter, H. Phys. Rep. 1985, 117, 265-387.

2. Lisse, C. M.; Dennerl, K.; Englhauser, J.; Harden, M.; Marshall, F. E.; J. Mumma, M.; Petre, R.; Pye, J.; Ricketts, M. J.; Schmitt, J.; Truemper, J.; West, R. G. Science 1996, 274, 205.

3. Wargelin, B. J. ; Drake, J. J. Astrophys. J. 2001, 546, L57.

4. Barany, A; Astner, G; Cederquist, H; Danared, D; Huldt, S; Hvelplund, P; Johnson, A; Knudsen, H; Liljeby, L; Rensfelt, K. G. Nucl. Instrum. Methods B 1985, 9, 397.

5. Dijkkamp, D.; Ciric, D.; Vlieg, E.; de Boer, A.; de Heer, F. J. J. Phys. B 1986, 18, 4763.

6. Ullrich, J.; Moshammer, R.; Dörner, R.; Jagutzki, O.; Mergel, V.; Schmidt-Böcking, H.; Spielberger, L. J. Phys. B 1997, 30, 2917.

7. Cassimi, A; Duponchel, S; Fléchard, X.; Jardin, P.; Sortais, P.; Hennecart, D. ; Olson, R. E. Phys. Rev. Lett. 1996, 76, 3679.

8. Niehaus, A. J. Phys. B 1986, 19, 2925.

9. Ostrovsky, V. N. J. Phys. B 1991, 24, 4553.

10. Janev, R. K.; Presnyakov, L. P.; Shevelko, V. P. Physics of Highly Charged Ions 1985 (SpringerVerlag: Heidelberg).

11. Harel, C.; Jouin, H. J. Phys. B 1992, 25, 221.

12. Barat, M.; Roncin, P. J. Phys. B 1992, 25, 2205.

13. Moretto-Capelle, P.; Bordenave-Montesquieu, A.; Gonzalez, A.; Benhenni, M. J. Phys. B 1994, 27, L317.

14. Frémont, F.; Merabet, H.; Chesnel, J.-Y.; Husson, X.; Lepoutre, A.; Lecler, D.; Stolterfoht N. Phys. Rev. A 1994, 50, 3117.

15. Chesnel, J.-Y.; Merabet, H.; Frémont, F.; Cremer, G.; Husson, X.; Lecler, D.; Rieger, G.; Spieler, A.; Grether, M.; Stolterfoht, N. Phys. Rev. A 1996, 53, 4198.

16. Chen, Z.; Shingal, R.; Lin, C. D. J. Phys. B 1991, 24, 4215.

17. Fritsch, W.; Lin, C. D. Phys. Rev. A 1992, 45, 6411.

18. Chesnel, J.-Y.; Frémont, F.; Sulik, B.; Ruiz-Méndez, C.; Merabet, H.; Bedouet, C.; Husson, X.; Grether, M.; Stolterfoht, N. Nucl. Instr. and Methods B 1999, 154, 142.

19. Fléchard, X; Harel, C; Jouin, H; Pons, B; Adoui, L; Frémont, F; Cassimi, A; Hennecart, D. J. Phys. B 2001, 34, 2759.

20. Sanchez, I; Bachau, H. J. Phys. B 1995, 28, 795.

21. Vaeck, N.; van der Hart, H. W.; Hansen, J. E. J. Phys. B 1995, 28, 5207.

22. Stolterfoht, N.; Sommer, K.; Swenson, K.; Havener, C. C.; Meyer, F. W. Phys. Rev. A 1990, 42, 5396.

23. Frémont, F.; Sommer, K.; Lecler, D.; Hicham, S.; Boduch, P.; Husson, X.; Stolterfoht, N. Phys. Rev. A 1992, 46, 222.

24. Bordenave-Montesquieu, A.; Moretto-Capelle, P; The Physics of Electronic and Atomic Collisions; AIP Conference Proceedings; Dubé, L. J.; Mitchell, J. B. A.; McConkey, J. W.; Brion, C. E.; Whistler, Canada, 1995.

25. Shah, M. B.; Gilbody, H. B. J. Phys. B 1990, 23, 1491.

26. McLaughlin, T. K.; McCullough, R. W.; Gilbody, H. B. J. Phys. B 1992, 25, 1257.

27. Meng, L.; Olson, R. E.; Folkerts, H. O.; Hoekstra, R. J. Phys. B 1994, 27, 2269. 
28. Folkerts, H. O.; Hoekstra, R.; Morgenstern, R. Phys. Rev. Lett. 1996, 77, 3339.

29. Ben-Itzhak, I.; Wells, E.; Stöckli, M. P.; Tawara, H.; Carnes, K. D. Phys. Scripta 1997, T73, 270.

30. Tarisien, M.; Adoui, L.; Frémont, F.; Cassimi, A. Phys. Scripta 1999, T80, 182.

31. Frémont, F.; Bedouet, C.; Chesnel, J.-Y.; Adoui, L.; Cassimi, A.; Tarisien, M. J. Phys. B 2000, 33, L249.

32. Fléchard, X; Duponchel, S; Adoui, L; Cassimi, A; Roncin, P; Hennecart, D. J. Phys. B 1997, 30, 3697.

33. Fléchard, X; thesis, Université de Caen, 1999 (unpublished).

34. Harel, C.; Jouin, H. J. Phys. B 1992, 25, 221.

35. Harel, C.; Jouin, H.; Pons, B. J. Phys. B 1991, 24, L425.

36. Stolterfoht, N. Z. Phys. 1971, 248, 81, 92.

37. Bedouet, C; thesis, Université de Caen, 1999 (unpublished).

38. Chen, L; Bernard, J; Martin, S; Denis, A; Désesquelles, J. Phys. Rev. A 1996, 54, 3049.

39. Cowan, R. D. The theory of Atomic Structure and Spectra, University of California Press: Berkley, 1981.

40. Merabet, H.; Cremer, G.; Frémont, F.; Chesnel, J.-Y.; Stolterfoht, N. Phys. Rev. A 1996, 54, 372.

41. Harel, C.; and Jouin, H. Europhys. Lett. 1990, 11,121.

42. Salin, A. Comput. Phys. Commun. 1991, 62, 58.

43. Frémont, F.; Bedouet, C.; Chesnel, J.-Y.; Husson, X. Phys. Rev. A 1998, 57, 4379.

44. DuBois, R. D.; Ali, I.; Cocke, C. L.; Feeler, C. R.; Olson, R. E. Phys. Rev. A 2000, 62, 060701R.

45. Graham, W. G.; Latimer, C. J.; Browning, R.; Gilbody, H. B. J. Phys. B 1974, 7, L405.

46. Tarisien, M.; Adoui, L.; Frémont F.; Lelièvre, D.; Guillaume, L.; Chesnel, J.-Y.; Zhang, H.; Dubois, A.; Mathur, D.; Kumar, S.; Krishnamurthy, M.; Cassimi, A. J. Phys. B 2000, 33, L11.

47. Sobocinski, P.; Rangama, J.; Chesnel, J.-Y.; Tarisien, M.; Adoui, L.; Cassimi, A.; Husson, X. ; Frémont, F. J. Phys. B 2001, 34, L367.

48. Wood, C. J.; Olson R. E. Phys. Rev. A 1999, 59, 1317.

49. Sobocinski, P.; Rangama, J.; Chesnel, J.-Y.; Tarisien, M.; Adoui, L.; Cassimi, A.; Husson, X. ; Frémont, F. XVIth International Conference on Application of Accelerators in Research and Industry, Denton, Texas, 2000. AIP Conference Proceedings, Duggan, J. L.; Morgan, I. L., Eds; 2001, p.114.

(C) 2002 by MDPI (http://www.mdpi.org), Basel, Switzerland. 